\section{International Scientific Journal Theoretical \& Applied Science}

\author{
p-ISSN: 2308-4944 (print) e-ISSN: 2409-0085 (online) \\ Year: $2015 \quad$ Issue: $11 \quad$ Volume: 31 \\ Published: $30.11 .2015 \quad$ http://T-Science.org
}

Elnur Latif oglu Hasanov Corresponding member of International Academy of Theoretical and Applied Sciences, Ph.D., Senior specialist of Ganja Department Azerbaijan National Academy of Sciences, Ganja, Azerbaijan 1-hasan@hotmail.com

SECTION 12. Geology. Anthropology. Archaeology.

\title{
GANJA IMAMZADE MAUSOLEUM AS A HISTORIC-CULTURAL SOURCE
}

Abstract: Basic features of Ganja Imamzade mausoleum have been investigated on the basis of different scientific sources and innovative methods.

Key words: Ganja, innovative research methods, historical-ethnographic research, Azerbaijan, Imamzade.

Language: French

Citation: Hasanov EL (2015) GANJA IMAMZADE MAUSOLEUM AS A HISTORIC-CULTURAL SOURCE. ISJ Theoretical \& Applied Science 11 (31): 67-69.

Soi: http://s-o-i.org/1.1/TAS-11-31-12 Doi: crossef http://dx.doi.org/10.15863/TAS.2015.11.31.12

\section{LE MAUSOLEE IMAMZADE DE GANDJA COMME UNE SOURCE HISTORIQUE ET CULTURELLE}

Résumé: Les caractéristiques de base de Ganja Imamzade mausolée ont été étudiés sur la base de différentes sources scientifiques et des méthodes novatrices.

Mots-clés: Gandja, méthodes de recherche innovants, recherche historique et ethnographique, Azerbaïdjan, Imamzade.

\section{Introduction}

La protection du patrimoine culturel, la restauration des monuments architecturaux, la protection de nos valeurs religieuses et morales (comme la politique primordiale d'Etat) ont été instituées par notre leader national Heydar Aliyev.

Les nombreux décrets et les ordonnances du Président de la République d'Azerbaïdjan, Ilham Aliyev, qui continue aujourd'hui avec succès la politique de raisonde notre leader national etles projets réalisés sous le patronagede l'ambassadricede bonne volonté de l'UNESCO et d'ISESCO Son Excellence la Première Dame d'Azerbaïdjan, Madame Mehriban Aliyéva, sont les manifestations exemplaires de la politique de protection de notre patrimoine culturel.

La protection des différents monuments historiques, culturels et religieux est une des priorités essentielles d'une politique d'Etat fermement enracinée dans le multiculturalisme et la tolérance.

La construction et la reconstruction de l'un des précieux monuments architecturaux et culturels de l'ancienne ville de Gandja, le mausoléeImamzadé, dans le style architectural de Gandja, a été réaliséeselonles sept décrets du Président azerbaïdjanais Ilham Aliyev entre 2010 et 2015.

\section{Materials and methods}

Le mausolée Imamzadéqui se trouve dans l'enceinte du Parc de la réserve historique et culturelle de Gandja - l'un des anciens centres scientifiques et culturels d'Azerbaïdjan - a été édifié en 739 sur le tombeau de Movlana Ibrahim, fils du cinquième Imam Mahomet Baguir et d'Ummu Hekim Mugeyra qizi Séguéfi, petit-fils de l'Imam Ali ibn Husseyn et arrière-petit-fils du Prophète Mahomet.

L'étymologie du mot «Imamzadé» signifie «l'enfant de l'Imam» ou encore «qui appartient à la génération de l'Imam».

Suite à la mort tragique de l'Imam Ali (Aleyhisselam) (661-750), la poursuite d'Ahli-beyt (les partisants d'Ali) au temps du pouvoir des Emévites obligeceux-ci à trouver refuge aux confins de Khilafat.

Dans le but de mettre fin à cette poursuite, Hazret Ibrahim (Aleyhisselam) émigra à Gandja, l'un des anciens centres de la culture islamique.

L'Imam Baguirmourut à la Mecqueau début de l'époque musulmane et fut enterré au cimetière de Bégui, auprès de ses parents. Son fils, Hazret Ibrahim fut, quant à lui, enterré à Gandja. 
Le mausolée construit au VIIIème siècle sur le tombeau de Hazret Ibrahim a été agrandi aux XIVième et XVème siècles, alors que la plupart des constructions qui l'entourent ont été édifiées entre les XVIIème et XVIIIème siècles.

Le mausolée est le monument le plus précieux du complexe Imamzadé. Sa hauteur est de $12 \mathrm{~m}$, celle du dôme est de $2,7 \mathrm{~m}$, et le diamètre est de $4,4 \mathrm{~m}$. Le dôme est recouvert de briques vernies.

Dans la culture populaire, on appelle Imamzadé« Göy Imam » soit «Imam Bleu». La présentation de ce lieu saint comme «Göy Imam» est liée à l'utilisation par les séïdesde la couleur bleue symbolique de la croyance du peuple azerbaïdjanais en un «Dieu Bleu» et symbolique du respect au «Göytürk» (Turc bleu).

$\mathrm{Au}$ XXième siècle, un important fait historique a été découvert grâce aux inscriptions présentes sur les pierres des murs intérieurs du mausolée.

De célèbres historiens-chercheurs, dont le défunt archéologue, professeur et docteur IsagDjafarzadé et la savante spécialiste de l'épigraphie, membre de l'Académie Nationale des Sciences de l'Azerbaïdjan, l'académicienne Méchédikhanoum Nématova, ont déchiffré ces inscriptions comme suit:

«Lui, Allah, est éternel. C'est la Terre Sainte, le paradis de Mevlana Ibrahim, le fils de l'Imam Mahomet Baguir (Dieu le salue). Il est mort 120 ans après le départ de son grand-père. Que Dieu le bénisse.»

\section{Conclusion and Recommendations}

Après une recherche approfondie faite sur ces inscriptions, il fut découvert que le monument a été élevé sur le tombeau de l'enfant de l'Imam en 738739 après J.-C.

Le territoire du complexe Imamzadéfaisait parti des biens des Sheïkhzamanov, considérés comme les représentants de la descendance de Nizami Gandjavi, le grand poète et penseur azerbaïdjanais.

Le dernier Khan de Gandja, Djavad khan Ziyadoglou (1748-1804), considéré comme la fierté de l'histoire de l'héroïsme en Azerbaïdjan, avait ordonné d'effectuer un grand travail de restauration à l'intérieur du mausolée Imamzadé.

Dans les années 1878-1879, sur l'initiative du général-major Israfil bey Yadigarzade, un travail de rénovation indispensable fut réalisé dans le complexe Imamzadé.

Grâce à la charité d'un groupe d'intellectuels de Gandja, le sanctuaire fut restauré au début du XXième siècle.

Les représentants de familles illustres de Gandja comme lesSheïkhzamanov, les Pishnamazzadé, Mirzé Mehdi Nadji, Mir Abbas aga (représentant de la branche des séïdes), le général-major Israfil bey Yadigarzadé et autres grandes personnalités ont été enterrés dans ce sanctuaire.

Durant des siècles, le complexe Imamzadé a été connu comme étant un lieu d'asile pour les sans-abris et lieu principal de la bienfaisance désintéressée.

Le mausolée Imamzadé était l'unique sanctuaire musulman qui fonctionnait librement sur le territoire de l'URSS.

Sous la domination soviétique, dans les années 1930-1944, le sanctuaire fut fermé, les constructions du complexe étant utilisées comme lieu d'accueil pour les enfants.

Cet endroit saint ouvrit ainsi ses bras aux petits enfants touchés par la guerre, qu'ils soientrusses ou d'autres nationalités.

Aujourd'hui, le complexe Imamzadé est considéré non seulement comme un sanctuaire saint $\mathrm{du}$ monde islamique, mais aussi comme un endroit saint pour les pèlerins étrangers.

\section{References:}

1. Balayev M (1991) İslam və onun ictimai-siyasi həyatda rolu. Bak1, 1991.

2. Ofəndiyev RS (1966) Azərbaycanın bədii sənətkarlığı. Bakı: Azərnəşr.

3. Ohmədov FM (2007) Gəncənin tarix yaddaş1. Gəncə: Elm.

4. Fətullayev-Fiqarov Ş (2003) Azərbaycanın qədim məscidləri. Azərbaycan-İrs jurnalı. №14-15.

5. Hasanov EL (2014) Innovative basis of research of local handicraft branches of Ganja of the second half of XIX - beginning of XX centuries. Mediterranean Journal of Social Sciences, vol. 5, № 23, Part IV, pp. 2359-2362. Doi:10.5901/mjss.2014.v5n23p2359

6. Hasanov EL (2014) To the question on the research of typical features of craftsmanship heritage of Ganja of the late XIX - early XX centuries. ISJ Theoretical \& Applied Science 12(20): 27-30. doi: http://dx.doi.org/10.15863/TAS.2014.12.20.7

7. Həsənov EL (2010) Gəncə İmamzadə türbəsində dulusçuluq elementləri. AMEA 


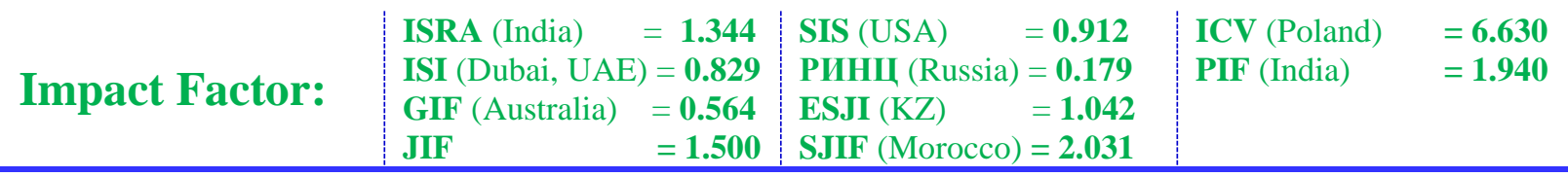

Gəncə Regional Elmi Mərkəz, Xəbərlər məcmuəsi. №42.

8. Həvilov HA (1991) Azərbaycan etnoqrafiyas1. Bakı: Elm

9. Qasimov M, Quliyev H (1986) Qədim xalq boyama üsulları. Qobustan, № 1 (69), Bakı.

10. Məmmədov FN (1976) XIX əsrdə Gəncə şəhərinin ərazisi, əhalisi və idarəsi (1868-ci ilə qədər). Azərbaycan SSR Elmlər
Akademiyasının Xəbərləri (Tarix, fəlsəfə və hüquq seriyas1), №3, s. 30-37.

11. Məmmədzadə K (1978) Azərbaycanda inşaat. Bakı: Elm.

12. Nishiaki Y, Hasanov EL (2014) About ethnoarchaeological and anthropological research of some prehistoric monuments of Ganja. ISJ Theoretical \& Applied Science, 1: 45-48.

doi: 